\title{
Impact of Cardiac Resynchronization Therapy on Indirect Inflammatory Markers
}

\author{
\begin{tabular}{l} 
Metin Çoksevim $^{\mathbf{1}}$ (D) Mustafa Yenerçağ \\
\hline 1 Ondokuz Mayis University Medical Faculty, Department of Cardiology, Samsun, Turkey. \\
2 Ordu University Medical Faculty, Department of Cardiology, Ordu, Turkey.
\end{tabular} \\ 3 Samsun Training and Research Hospital, Department of Cardiology, Samsun, Turkey.
}

\section{Onur Osman Şeker ${ }^{3}$ (D)}

\begin{abstract}
Background: Cardiac resynchronization therapy (CRT) is an established treatment for patients with symptomatic chronic heart failure with reduced ejection fraction (HFrEF) and prolonged QRS despite optimal pharmacological therapy. Inflammation plays a crucial role in the pathogenesis and progression of cardiovascular disease. The role of CRT pre-implantation inflammatory condition assessed using routine laboratory tests has been rarely investigated.

In this study we aimed to evaluate the effect of CRT on indirect inflammatory markers such as neutrophil-to-lymphocyte ratio (NLR), platelet-to-lymphocyte ratio (PLR) and lymphocyte-to- the monocyte (LMR) ratio.

Methods: 75 CRT patients were included in the study retrospectively. Before the CRT implantation, clinical and demographic data were recorded from all patients. NLR, PLR and LMR ratio were measured before CRT implantation. The patients were reevaluated minimum six months after CRT; the above-mentioned parameters were measured again and compared to the pre-CRT period.

Results: Compared to the period before CRT, laboratory findings such as white blood cell $(3.5 \pm 2.2103 \mathrm{uL}$ vs. $3.2 \pm 2.4103 \mathrm{uL} ; \mathrm{p}=$ 0.006), neutrophyl ( $1.9 \pm 0.4103 \mathrm{uL}$; vs. $1.4 \pm 0.4103 \mathrm{uL} ; \mathrm{p}=0.002)$, NLR (3.8 $\pm 0.3103 \mathrm{uL}$; vs. $1.7 \pm 0.1103 \mathrm{uL} ; \mathrm{p}<0.001)$, PLR $(490.2$ $\pm 199103 \mathrm{uL}$; vs. $381 \pm 105103 \mathrm{uL} ; \mathrm{p}<0.001)$ levels were significantly lower after 6 months of CRT implantation. Lymphocyte counts $(0.5 \pm 0.3103 \mathrm{uL}$ vs. $0.8 \pm 0.2103 \mathrm{uL} ; \mathrm{p}=0.001)$ were significantly higher in the post CRT group. A significant and positive correlation of the reduction in NLR ( $r s=0.362, p=0.001$ ) and PLR ( $r s=0.562, p<0.001$ ) was found with the increased six minute walking test (6-MWT).
\end{abstract}

Conclusion: The NLR, PLR and MLR were decreased after CRT implantation. The modest decrease in these parameters demonstrates the effect of restoring the heart's electromechanical synchrony after CRT on inflammation.

Key Words: Congestive Heart Failure, Cardiac Resynchronization Therapy, İnflammatory Markers. 


\section{INTRODUCTION}

Approximately $1-2 \%$ of the adult population in developed countries have heart failure (HF), with the prevalence rising to $\geq 10 \%$ among persons 70 years of age or older (1). HF can be classified into three types based on the condition of left ventricular (LV) systolic function: HF with preserved ejection fraction $(\mathrm{EF})(\mathrm{EF} \geq 50 \%)$, mid-range $\mathrm{HF}(\mathrm{EF}: 40$ - 49 $\%)$ and HF with reduced ejection fraction (HFrEF) $(\mathrm{EF}<$ $40 \%$ ) (2) . Cardiac resynchronization therapy (CRT) is an established treatment for patients with symptomatic chronic HFrEF and prolonged QRS despite optimal pharmacological therapy.

By restoring the heart's electromechanical synchrony, CRT improves self-reported symptoms and reduces mortality and rehospitalization for heart failure (3). Unfortunately, almost a third of patients do not respond favourably to CRT (4). Several characteristics are associated with improved response, and thus survival following CRT implantation (5)

Inflammation plays a crucial role in the pathogenesis and progression of cardiovascular disease. Numerous inflammatory biomarkers are correlated with disease severity and prognosis across throughout HF (6). However, the role of CRT pre-implantation inflammatory condition assessed using routine laboratory tests has been rarely investigated.

In this study we aimed to evaluate the effect of CRT on indirect inflammatory markers such as neutrophil-tolymphocyte ratio (NLR), platelet-to-lymphocyte ratio (PLR) and lymphocyte-to- the monocyte (LMR) ratio.

\section{MATERIALS AND METHODS}

This study was approved by the Ondokuz Mayıs University institutional ethics committee in which it took place in line with the recommendations of the Declaration of Helsinki (Date: 25.02.2016 Decision no: 2016-90).

\section{Study Population and Data Collection}

Subjects consisted of 82 consecutive patients undergoing CRT, between January 2017 and December 2019, at Ondokuz Mayıs University School of Medicine Cardiology Department who were retrospectively enrolled into the study. Patients were included according to following criteria: (1) chronic HF with reduced LVEF ( $\leq 35 \%)$ and (2) prolonged QRS interval ( $\geq 130 \mathrm{msn}$ ) with LBBB morphology (3) have indication for CRT implantation according to the 2016 European Society of Cardiology guideline for the diagnosis and treatment of acute and chronic HF. CRT implantation was performed to all participants.

Patients with mechanical tricuspid valve, recent myocardial infarction or coronary artery bypass graft surgery ( $\leq$ six months), decompensated $\mathrm{HF}$, malignancies, chronic inflammatory disease, haematological disorders, renal or hepatic disorders, right bundle branch block morphology on electrocardiogram (ECG), right ventricular pacing only, pacemaker upgraded to CRT, LV lead inserted into other than lateral or postero-lateral branches of coronary sinus, life expectancy of less than 12 months, and followup interval less than six months were excluded from the study. Thus, 7 patients were excluded, and the study cohort included a total of 75 patients. All patients included in the study with either sinus rhythm or atrial fibrillation provided biventricular pacing over $90 \%$.

An independent physician who was blinded to all other data performed the clinical evaluation, including assessment of New York Heart Association (NYHA) class, in all of the patients. QRS duration was measured by surface ECG using the widest QRS complex from the II, V1, and V6 leads. All patients were evaluated in terms of age, gender, coronary artery disease history, diabetes mellitus, hyperlipidaemia, hypertension, and other concomitant diseases. Patients were classified as ischaemic or nonischaemic aetiology of HF. The patients underwent a detailed echocardiographic examination at baseline and six months after the CRT.

\section{Echocardiography}

Transthoracic echocardiography (TTE) was performed by an experienced echocardiography specialist who was blinded to other data. Vivid E9 (GE Vingmed Ultrasound, Horten, Norway) TTE device and M5S (1.5-4.5 MHz) ultrasound probe were used for the echocardiographic measurements. Left ventricular end-systolic volume (LVESV), left ventricular end-diastolic volume (LVEDV), left ventricle end-diastolic (LVEDD) and end-systolic diameters (LVESD), and left atrium (LA) anteroposterior diameter were measured from the long axis view of the heart using TTE. Ejection fraction (EF) was calculated by Modified Simpson method using apical 4-chamber and 2-chamber images. Valvular heart pathologies were detected and graded. Pulmonary arterial pressure was measured.

\section{CRT implantation}

Following left pectoral region incision, subclavian venous puncture was performed, and right ventricle and right 
atrium leads were placed. After this, coronary sinus was found using a left amplatz catheter, and images were recorded using a contrast-enhancing agent for the selection of the suitable branch. LV lead was placed on the lateral or posterolateral branch of coronary sinus if possible. All electrodes were connected to the generator, and the pouch was closed following stimulus and threshold values were controlled. After implantation AV delay of the patients was set to be $120 \mathrm{~ms}$, and VV delay was $0 \mathrm{~ms}$ for optimal resynchronization.

\section{Laboratory measurements}

In our hospital, blood samples were collected from the antecubital vein within 24 hours of hospital admission. Complete blood cell counts including total white blood cell (WBC), platelet, neutrophil, lymphocyte, and monocyte counts, and haemoglobin level were all measured with an autoanalyzer. NLR was calculated by dividing the neutrophil count by the lymphocyte count. PLR was calculated by dividing the platelet count by the lymphocyte count. LMR was calculated by dividing the lymphocyte count by the monocyte count. Venous blood samples were obtained without venostasis by venepuncture of the large antecubital veins of the patients at least $24 \mathrm{~h}$ before CRT implantation and were immediately studied in the laboratory without any time delay. Study patients were reevaluated minimum 6 months after the CRT implantation and the parameters of pre-CRT and post-CRT periods were compared eachother.

\section{Definitions}

Ischemic cardiomyopathy and non-ischemic cardiomyopathy definitions were made based on the presence or absence of myocardial infarction events or $75 \%$ or more stenosis in the left coronary artery.

\section{STATISTICAL ANALYSIS}

Statistical analyses were performed using SPSS 22 for Windows (SPSS Inc., Chicago, IL, USA). The continuous variables were tested for a normal distribution using the Kolmogorov-Smirnov test. Normality was checked using the Shapiro-Wilk statistic test. Normally distributed data were presented as the mean \pm standard deviation and non-normally distributed data as the median with an interquartile range. The categorical variables were expressed as percentages. A paired sample $t$ test or Wilcoxon's signed-rank test was performed according to the normality of the clinical variables to compare clinical parameters before and six mounth after the CRT. Spearman correlation analysis was used for variables not showing normal distribution. The NYHA class change was compared using Cochran Mantel-Haenszel test for ordered variables. Spearman correlation analysis was performed to examine the relationship between change in NLR, PLR and six-minute walking test (6-MWT). Statistical significance was set at $\mathrm{p}<0.05$.

\section{RESULTS}

A total of 75 CRT patients were included in the study. Of the study patients, $54 \%$ were males; the mean age was 63.1 \pm 12.5 years, and $64 \%$ had ischemic etiology. Hypertension was present in $80 \%$ of patients. Therapy with betablockers and diuretics were at high rates $(85.3 \%$ vs $96 \%$, respectively). The basic clinical features and laboratory parameters of the study groups are listed in Table 1. The echocardiogram and laboratory findings and the clinical parameters evaluated before CRT and after six months are shown in Table 2. The heart rate $(72.9 \pm 4.9 \mathrm{bpm}$ vs $62.8 \pm 6.5 \mathrm{bpm}, \mathrm{p}=0.002)$. The LVESV, LVEDV, LVESD and LVEDD decreased significantly $(\mathrm{p}<0.05)$. While significant increases occurred in LVEF ( $30.6 \pm 2.9 \%$ vs $31.9 \pm 2.5 \%$, p $<0.001)$ and the cardiac index $\left(2.3 \pm 0.4 \mathrm{~L} / \mathrm{min} / \mathrm{m}^{2}\right.$ vs 2.5 $\pm 0.5 \mathrm{~L} / \mathrm{min} / \mathrm{m}^{2}, \mathrm{p}<0.001$ ), no significant changes were observed in the mitral regurgitation figure ( $\geq$ moderate) (31 vs $27, \mathrm{p}=0.288$ ). The patients exhibited significant NYHA classes improvement following the initiation of CRT. 6-MWT significantly increased after 6 months of CRT implantation $(256 \pm 42$ vs. $296 \pm 52 ; \mathrm{p}=0.002$. In their laboratory findings white blood cell $\left(3.5 \pm 2.210^{3}\right.$ uL vs. $\left.3.2 \pm 2.410^{3} \mathrm{uL} ; \mathrm{p}=0.006\right)$, neutrophyl $\left(1.9 \pm 0.410^{3} \mathrm{uL}\right.$; vs. $\left.1.4 \pm 0.410^{3} \mathrm{uL} ; \mathrm{p}=0.002\right), \operatorname{NLR}\left(3.8 \pm 0.310^{3} \mathrm{uL} ;\right.$ vs. 1.7 $\left.\pm 0.110^{3} \mathrm{uL} ; \mathrm{p}<0.001\right)$, PLR $\left(490.2 \pm 19910^{3} \mathrm{uL} ;\right.$ vs. $381 \pm$ $\left.10510^{3} \mathrm{uL} ; \mathrm{p}<0.001\right)$ levels were significantly lower after 6 months of CRT implantation. Lymphocyte counts $(0.5 \pm$ $0.310^{3}$ uL vs. $0.8 \pm 0.210^{3} \mathrm{uL} ; \mathrm{p}=0.001$ ) were significantly higher in the post CRT group. In addition, there was no difference between the groups in terms of other laboratory findings and LMR ( $p>0.05)$.

A significant and positive correlation of the reduction in NLR was found with the increased 6-MWT ( $\mathrm{rs}=0.362, \mathrm{p}$ $=0.001$ ) (Fig.1A). A significant and positive correlation of the reduction in PLR was found with the increased 6-MWT ( $r s=0.562, p<0.001$ ) (Fig.1B). 
Table 1. Baseline demographic and clinical parameters of the study population

\begin{tabular}{|c|c|}
\hline Variable & $(\mathrm{n}=75)$ \\
\hline Age (years) & $63.1 \pm 12.5$ \\
\hline Gender & \multirow{3}{*}{$\begin{array}{l}41(54.6) \\
34(45.3)\end{array}$} \\
\hline Men, n (\%) & \\
\hline Women, n (\%) & \\
\hline Body mass index $\left(\mathrm{kg} / \mathrm{m}^{2}\right)$ & $22.6 \pm 2.6$ \\
\hline Smoking & $26(34.6)$ \\
\hline \multicolumn{2}{|l|}{ Etiology of heart failure } \\
\hline Ischemic & $48(64)$ \\
\hline Non-Ischemic & $27(49)$ \\
\hline \multicolumn{2}{|l|}{ NYHA class, n (\%) } \\
\hline II & $25(33.3)$ \\
\hline III & $39(52)$ \\
\hline IV & $11(14.6)$ \\
\hline Hypertension, n (\%) & $60(80)$ \\
\hline Diabetes mellitus, $\mathrm{n}(\%)$ & $28(37.3)$ \\
\hline Atrial fibrillation, $\mathbf{n}(\%)$ & $18(24)$ \\
\hline Beta-blocker, n (\%) & $64(85)$ \\
\hline ACEI or ARB, $n$ & $67(89)$ \\
\hline ARNI, $\mathbf{n}$ & $5(6)$ \\
\hline Aldosterone receptor blocker, n (\%) & $51(68)$ \\
\hline Diuretic, n (\%) & $72(96)$ \\
\hline Ivabradine, n (\%) & $30(40)$ \\
\hline Digoxin, n (\%) & $16(21)$ \\
\hline ECG branch block & $124.2 \pm 9.1$ \\
\hline LBBB, $\mathbf{n}$ & $61(81.4)$ \\
\hline Other bransch blocks, $\mathbf{n}$ & 14 (18.6) \\
\hline
\end{tabular}

Numerical variables are presented as mean \pm SD and categorical variables as percentages. NYHA: New York Heart Association; ACEI: Angiotensin-converting enzyme inhibitor; ARB: Angiotensin receptor blocker; ECG: Electrocardiography; LBBB: Left Bundle Branch Block
Table 2. Echocardiographic, laboratory and clinical parameters before and six month after CRT

\begin{tabular}{|c|c|c|c|}
\hline Parameters & Baseline & 6rd month & p value \\
\hline Heart rate (bpm) & $72.9 \pm 4.9$ & $62.8 \pm 6.5$ & 0.002 \\
\hline QRS duration, msn & & & \\
\hline Creatinine, $\mathrm{mg} / \mathrm{dL}$ & $1.01 \pm 0.26$ & $1.14 \pm 0.28$ & 0.098 \\
\hline Potassium, mEq/L & $4.1 \pm 0.2$ & $4.4 \pm 0.3$ & 0.097 \\
\hline NYHA class & & & $<0.001$ \\
\hline I n, $(\%)$ & 0 & $3(4)$ & \\
\hline II n, $(\%)$ & $25(33.3)$ & $40(53.3)$ & \\
\hline III n, $(\%)$ & $39(52)$ & $25(33.3)$ & \\
\hline IV n, $(\%)$ & $11(14.6)$ & $7(9)$ & \\
\hline 6-MWT, m & $256 \pm 42$ & $296 \pm 52$ & 0.002 \\
\hline White blood cell, $10^{3} \mathrm{uL}$ & $3.5 \pm 2.2$ & $3.2 \pm 2.4$ & 0.006 \\
\hline Hemoglobin, g/dL & $10.4 \pm 2.6$ & $10.8 \pm 2.4$ & 0.058 \\
\hline Neutrophil, $10^{3} \mathrm{uL}$ & $1.9 \pm 0.4$ & $1.4 \pm 0.4$ & 0.002 \\
\hline Lymphocyte, $10^{3} \mathrm{uL}$ & $0.5 \pm 0.3$ & $0.8 \pm 0.2$ & $<0.001$ \\
\hline Monocyte, $10^{3} \mathrm{uL}$ & $0.6 \pm 0.3$ & $1 \pm 0.2$ & $<0.001$ \\
\hline Platelet, $10^{3} \mathrm{uL}$ & $245 \pm 43$ & $305 \pm 53$ & $<0.001$ \\
\hline NLR & $3.8 \pm 0.3$ & $1.7 \pm 0.1$ & $<0.001$ \\
\hline PLR & $490 \pm 199$ & $381 \pm 105$ & $<0.001$ \\
\hline LMR & $0.8 \pm 0.25$ & $0.8 \pm 0.21$ & 0.856 \\
\hline LVEDd, mm & $58(56-61)$ & $56(55-61)$ & 0.017 \\
\hline LVESd, mm & $44(42-47)$ & $41.5(40-45)$ & $<0.001$ \\
\hline LVEF, (\%) & $30.6 \pm 2.9$ & $31.9 \pm 2.5$ & $<0.001$ \\
\hline LVEDV, mL & $\begin{array}{l}161(146- \\
176)\end{array}$ & $\begin{array}{c}153.5(146- \\
167)\end{array}$ & 0.007 \\
\hline LVESV, mL & $\begin{array}{l}114(100- \\
125)\end{array}$ & $\begin{array}{c}100(96- \\
110)\end{array}$ & $<0.001$ \\
\hline $\begin{array}{l}\text { Cardiac Index (L/min/ } \\
\left.\mathrm{m}^{2}\right)\end{array}$ & $2.3 \pm 0.4$ & $2.5 \pm 0.5$ & 0.001 \\
\hline $\begin{array}{l}\text { Mitral insufficiency ( } \geq \\
\text { moderate) } n,(\%)\end{array}$ & $31(34)$ & $27(31.3)$ & 0.288 \\
\hline sPAP, mmHg & $33.9 \pm 3.7$ & $30.1 \pm 2.9$ & $<0.001$ \\
\hline
\end{tabular}


Numerical variables are presented as mean \pm SD and categorical variables as percentages. NYHA: New York Heart Association; 6-MWT: Six month walk test; NLR: neutrophil-lymphocyte ratio; PLR: Platelet-lymphocyte ratio; LMR: Lymphocyte-monocyte ratio; LV: Left ventricular; EF: Ejection fraction; LVEDd: Left ventricular end-diastolic diameter; LVESd: Left ventricular endsystolic diameter; LVEDV: Left ventricular end-diastolic volume; LVESV: Left ventricular end-systolic volume; sPAB: Systolic pulmonary arterial pressure.

Fig.1 (A) Correlation of change in 6-MWT with PLR. (B) Correlation of change in 6-MWT with change in NLR. rs: indicates correlation coefficient.
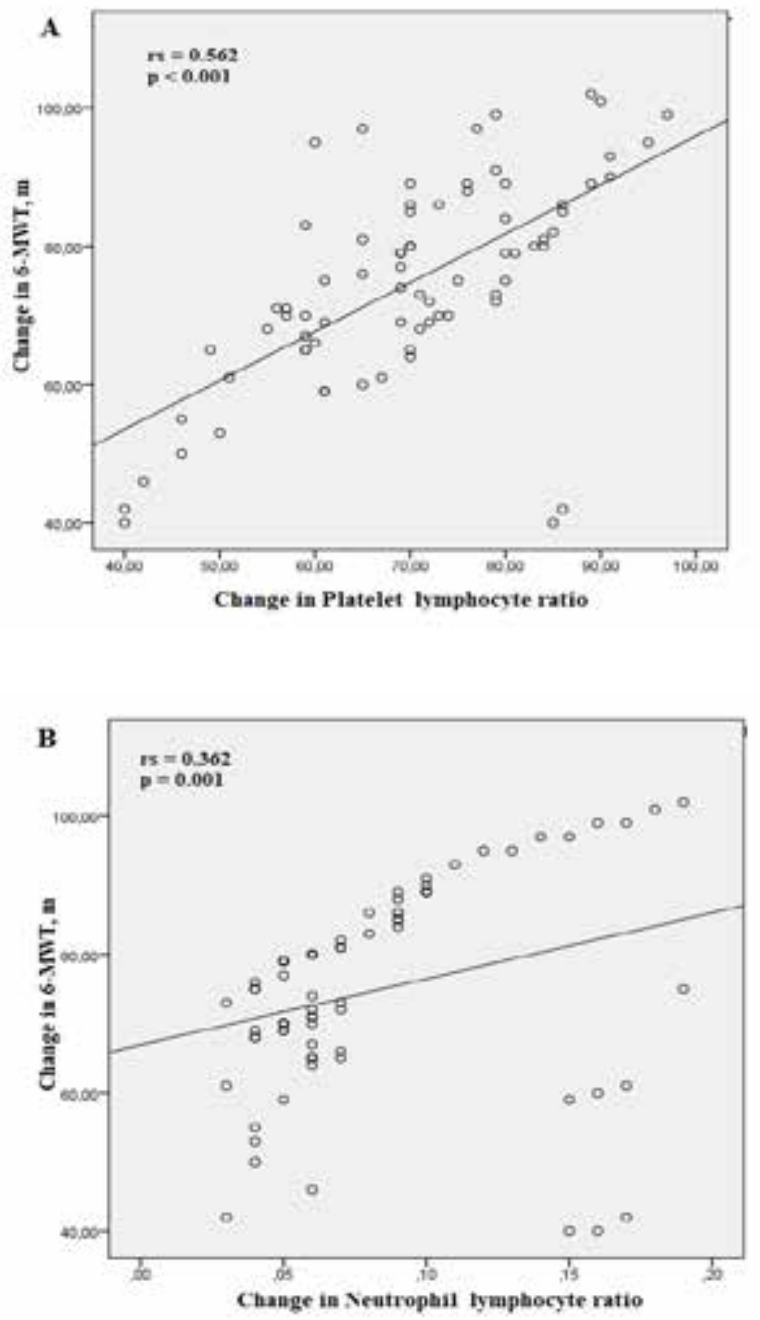

\section{DISCUSSION}

As a result of our study, we found that CRT causes a moderate decrease in indirect inflammatory parameters. These data suggest that decrease in inflammatory markers of which increased levels are associated with poor outcome in cardiovascular events, important in positive prognostic effects after CRT.

The prevalence of heart failure, especially with the decrease in deaths due to myocardial infarction and sudden cardiac death, is increasing worldwide (1). Endpoints have improved considerably with advances in HF treatment (2).

Cardiac resynchronization therapy, which has a significant contribution to the positive effects, increases cardiac performance in selected eligible patients and provides a significant reduction in symptoms and morbidity and mortality (3). In electrical and mechanical synchronization failure in HF patients, the right and LV leads placed with CRT create two ventricular activation waves that are distributed in opposite directions starting from where they are placed. The beneficial effect of these two depolarization waves is to synchronize the contraction of the LV walls. Thus, the performance of the myocardium, which starts to contract synchronously, increases with both the mechanical effect and the reversing remodelling effect (7).

Heart failure is a systemic condition with increased levels of inflammatory markers. Treatments targeting these pathways have shown a favourable prognostic effect in this syndrome (8).

It is known that the immune system and inflammation play an important role in the pathogenesis of HF. However, the effect of the immunological system on prognosis remains unclear (8). The basis of the interaction between leukocyte derivatives and HF is highly complex (9).

In summary, it has been suggested that systemic cytokine release, which potentially causes lymphocyte apoptosis and activation of the hypothalamic-pituitaryadrenal axis, causes a decrease in \% lymphocyte count, especially due to physical stress (8). Previous studies have shown that \% lymphocyte count is significantly associated with HF incidence, HF hospitalizations and mortality (10). 
Neutrophils play an important role in the inflammation process by producing myeloperoxidase, which promotes phagocytic function. Increased levels of this enzyme also cause excessive free radical production, which has detrimental effects on the myocardium (11). In this context, $\mathrm{Avc1}$ and his friends observed a significant negative correlation between NLR and LVEF in patients with idiopathic dilated cardiomyopathy. They found worse functional classes in patients with higher NLR levels in their study and concluded that the higher NLR was useful for evaluating the severity of HF (11). Yildiz and her friends reported higher NLR levels and decreased functional capacity in HFrEF patients with similar LVEF (12). Additionally, Agacdiken and his friends found that the basal NLR is a predictor of the response to CRT (13). Balc1 et al., in their study evaluating the response to CRT, found higher NLR and PLR values in patients who did not respond to CRT (14). In our study, a significant decrease in NLR, PLR and MLR was detected in all patient groups after CRT implantation. These results suggest that; the effectiveness of CRT can be demonstrated by using simple inflammatory markers.

This study has some limitations. First, this retrospective study was conducted in a single centre with a small sample size. Second, additional inflammation markers were not assessed to address the other confounding factors. Third the relationship between the inflammatory markers and clinical outcomes were not evaluated. A prospective randomized multi-center study with a larger study population might increase the significance of the presented results.

The NLR, PLR and MLR were decreased after CRT implantation. The modest decrease in these parameters demonstrates the effect of restoring the heart's electromechanical synchrony after CRT on inflamation. These results appeared to be associated with positive response to CRT.

\section{Acknowledgments}

We thank to Selim Çoksevim for English editing.

\section{Declarations}

The authors received no financial support for the research and / or authorship of this article. There is no conflict of interest.

This study was approved by the Ondokuz Mayıs University institutional ethics committee in which it took place in line with the recommendations of the Declaration of Helsinki (Date: 25.02.2016 Decision no: 2016-90).

\section{REFERENCES}

1. Degertekin M, Erol Ç, Ergene O, Tokgözoðlu L, Aksoy M, Erol MK, et al. Heart failure prevalence and predictors in Turkey: HAPPY study. Archives of the Turkish Society of Cardiology. 2012;40(4):298-308.

2. Ponikowski P, Voors AA, Anker SD, Bueno H, Cleland JG, Coats AJ, et al. 2016 ESC Guidelines for the diagnosis and treatment of acute and chronic heart failure: The Task Force for the diagnosis and treatment of acute and chronic heart failure of the European Society of Cardiology (ESC) Developed with the special contribution of the Heart Failure Association (HFA) of the ESC. European heart journal. 2016;37(27):2129-200.

3. Cleland JG, Abraham WT, Linde C, Gold MR, Young JB, Claude Daubert $\mathrm{J}$, et al. An individual patient meta-analysis of five randomized trials assessing the effects of cardiac resynchronization therapy on morbidity and mortality in patients with symptomatic heart failure. European heart journal. 2013;34(46):3547-56.

4. Van Bommel RJ, Bax JJ, Abraham WT, Chung ES, Pires LA, Tavazzi $\mathrm{L}$, et al. Characteristics of heart failure patients associated with good and poor response to cardiac resynchronization therapy: a PROSPECT (Predictors of Response to CRT) sub-analysis. European heart journal. 2009;30(20):2470-7.

5. Goldenberg I, Hall WJ, Beck CA, Moss AJ, Barsheshet A, McNitt S, et al. Reduction of the risk of recurring heart failure events with cardiac resynchronization therapy: MADIT-CRT (Multicenter Automatic Defibrillator Implantation Trial With Cardiac Resynchronization Therapy). Journal of the American College of Cardiology. 2011;58(7):72937.

6. Braunwald E. Biomarkers in heart failure. New England Journal of Medicine. 2008;358(20):2148-59.

7. Singh JP, Gras D. Biventricular pacing: current trends and future strategies. European heart journal. 2012;33(3):305-13.

8. Vaduganathan M, Greene SJ, Butler J, Sabbah HN, Shantsila E, Lip GY, et al. The immunological axis in heart failure: importance of the leukocyte differential. Heart failure reviews. 2013;18(6):835-45.

9. Maisel A, Knowlton K, Fowler P, Rearden A, Ziegler M, Motulsky H, et al. Adrenergic control of circulating lymphocyte subpopulations. Effects of congestive heart failure, dynamic exercise, and terbutaline treatment. The Journal of clinical investigation. 1990;85(2):462-7.

10. Cho JH, Cho H-J, Lee H-Y, Ki Y-J, Jeon E-S, Hwang K-K, et al. Neutrophillymphocyte ratio in patients with acute heart failure predicts in-hospital and long-term mortality. Journal of clinical medicine. 2020;9(2):557.

11. Tang WW, Tong W, Troughton RW, Martin MG, Shrestha K, Borowski A, et al. Prognostic value and echocardiographic determinants of plasma myeloperoxidase levels in chronic heart failure. Journal of the American College of Cardiology. 2007;49(24):2364-70.

12. Yıldız A, Yüksel M, Oylumlu M, Polat N, Akıl MA, Acet H. The association between the neutrophil/lymphocyte ratio and functional capacity in patients with idiopathic dilated cardiomyopathy. Anatolian journal of cardiology. 2015;15(1):13.

13. Agacdiken A, Celikyurt U, Sahin T, Karauzum K, Vural A, Ural D. Neutrophil-to-lymphocyte ratio predicts response to cardiac resynchronization therapy. Medical Science Monitor: International Medical Journal of Experimental and Clinical Research. 2013;19:373.

14. Balci KG, Balci MM, Sen F, Canpolat U, Akboga MK, Unal S, et al. The role of baseline indirect inflammatory markers in prediction of response to cardiac resynchronisation therapy. Kardiologia Polska (Polish Heart Journal). 2016;74(2):119-26. 